\title{
LA FAMILIA ACMAEIDAE (GASTROPODA, ARCHAEOGASTROPODA) EN EL PERU
}

\author{
Carlos Paredes Q.
}

Departamento de Ciencias Biológicas. Sección Zoología.

Universidad Nacional Mayor de San Marcos. Lima - Peni.

\section{SUMARIO}

Se reportan siete especies de la familia Acmaeidae de la zona litoral rocosa del Departa. mento de Lima: Scurria viridula (Lamarck), S. parasitica (Orbigny), S. variabilis (Sowerby), S. zebrina (Lesson), Collisella orbignyi (Dail), $C$. ceciliana (Orbigny) y $C$. araucana (Orbigny) Se amplía el lími te norte de la distribución geografica de las dos últimas especies y asimismo, para $\boldsymbol{S}$. zebrina y $\boldsymbol{S}$. parasitica.

Se informa sobre la zonación vertical de las especies, su dominancia relativa y otras observaciones ecológicas.

De la revisión de la literatura y los resultados del presente estudio, se concluye que, con. juntamente con Scurria scurra (Lesson, 1830), son ocho las especies de Acmaeidae presen. tes en la Provincia Peruana.

\section{MUMMRY}

This paper deal with seven species of Acmaeidae-Scuria whitula (Lamarck), S. parasitica (Orbigny), S. variabilis (Sowerby), S. zebrina (Lesson), Collisella orbignyi (Dall), $C$. ce. cillina (Orbigny) y $C$. araucani (Orbigny), reported along the rocky shore of Lima Department.

Information concerned with geographical and vertical distribution, and others ecological data are also added.

\section{INTRODUCCION}

Keen (1971) sefiala que los investigadores americanos han seguido la tendencia de incluir a todas las especies de Acmaeidae en el género Acmaea, sin considerar las diferencias que se presentan en la rádula. Este género se caracteriza porque la rádula presenta tres pares de dientes laterales de igual tamaño y carece de dientes marginales.

Aparte del clásico trabajo del Dall (1909) sobre la fauna malacológica peruana solo se conoen los estudios de Vegas (1968), quien reportó a Acmaea orbinyi y a Scurria scurra; y de Peffa $(1970,1972)$, quien reporta además de las especies citadas a Acmaea viridula y $A$. variabilis.

Uitumamente Marincovich (1973) ha realizado una revisión de esta tamilia, indicanao que falta estudiar material de la costa peruana. Este autor, estudiando la colección del Natural History Museum Los Angeles County (LACM) y material colectrdo por él, en Iquique, Chile, reporta ocho especies de la famuli Acmaeidae pertenecientes a los generos Scurria y Colli. sella, los cuales se diferencian por pesentar o no cordon branquial, respectivamente. En estos géneros la rádula presenta el par de dientes mediolaterales más desarrollados y un par de dientes marginales nudimentarios o uncini.

En este trabajo se ha prestado especial atención al estudio del cordón branquial, habiéndose determinado siete especies de Acmaeidae, y contribuido a la determinación genérica de Scumia noriablis (Sowerby, 1839) y S. zebrina (Lesson, 1830), especies que fueron consideradas por Marincovich (op. cit.) en el genero Collisella, debido a la falta de material preser. vado. 


\section{MATERIAL Y METODOS}

Re乞olecciones y observaciones esporádicas se han realizado durante varios affos por razones de enseñanza. En 1972 y parte de 1973 se estudió la zonación biocenológica, se determinó la distribución vertical de las especies y se obtuvieron los datos bioecológicos en los lugares de estudio indicados en la figura 1 .

La recolección se realizó a mano empleando espátula, cuchillo, recipientes de plástico, etc. Para la fijación se utilizó formol al 6 o/o neutralizado con borax.

La zonación vertical se basa en un esquema modificado de Stephenson y Stephenson (1949), recientemente aplicado por el autor del presente trabajo, en la orilla rocosa del Departamento de Lima. (Paredes, 1974).

La dominancia relativa fué apreciada utilizando las escalas ideadas por Crisp y Southward, Bellantine, y Moise y Nelson Smith (Tait, 1971).

Respecto a la sinonimia, se indican solamente.los nombres utilizados por Vegas (1968), Peǹa (1970, 1972) yMarincovich (1973).

\section{RESULTADOS}

Se confirmó que la totalidad de especies presentes en Iquique, existen en el Perú, ya que si bien, no se registró a Scurria scurra (Lesson, 1830), ello se debe a que esta especie vive en el nivel sublitoral, el cual no fue explorado; sin embargo, su presencia está comprobada por los reportes de Vegas (1968) y Peña $(1970,1972)$.

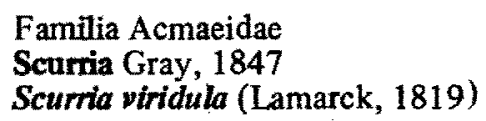

(Figura 2 - 3)

Acmaea viridula, Peña, 1970:155; 1972:40, fig.

Scurria viridula, Marincovich, 1973:23, fig. 40

Valva gruesa, de contorno casi redondeado y de color verdoso; escultura consiste en anchas costillas blanquecinas; el interior es blanco brilloso, con el area central manchada de pardo oscuro en los ejemplares juveniles; el margen es ligeramente festonado y de coloraración similar a la externa. Longitud, $39.5 \mathrm{~mm}$; ancho, $34 \mathrm{~mm}$; altura, $17.5 \mathrm{~mm}$.

Observaciones: Frecuente en el mediolitoral superior, sobre la superficie libre de las rocas; en los frentes muy expuestos llega hasta la franja supralitoral; algunos ejemplares presentan pequeños Chthamalus cirratus Darwin, epizoicos, y también a Scurria parasitica (Orbigny).

Distribución: Peña (1970), de Piura a Tacna; Marincovich (1973), Paita e Isla . Lobos, Perú a Valparaíso (Dall, 1909) y Punta Choros, Chile (420 S; Dell, 1971).

Localidades: Hallada en todes los lugares de estudio.

Scurria parasitica (Orbigny, 1841)

(Figura 4 - 5)

Scurria parasitica, Marincovich, 1973:21, fig. 37

Conchilla de base oval, poco elevada y esculpida con finas costillas radiales; externamen. te se presentan bandas radiales pardas y blanquecinas; interior pardo grisáceo con manchas marrones $y$ pardas en el área central, y con el margen coloreado según el patrón externo. Los ejemplares con esta coloración típica alcanzan hasta $8 \mathrm{~mm}$ de longitud; los de mayor talla alcanzan $15 \mathrm{~mm}$ de longitud, $6 \mathrm{~mm}$ de ancho y $6 \mathrm{~mm}$ de altura, presentando la mayor parte de la conchilla corroída. Pocos ejemplares de la forma oscura coffea (Marincovich, 1973) fueron encontrados, con una longitud de $12 \mathrm{~mm}$., color exterior pardo grisáseo uniforme sin bandas radiales, con el interior marrón azulado, y con el área central con manchas marrón claro.

Tanto Vegas (1968) como Peña (1972) consideran a esta especie sinónimo de Scurria 
scurra (Lesson, 1830), aparentemente sin haber estudiado a $S$, parasitica, ya que las ilustraciones que presentan corresponden a ejemplares de $S$. scurra.

Observaciones: Frecuente en el mediolitoral inferior y en la franja infralitoral de orillas expuestas, viviendo epizoico en excavaciones superficiales de Fissurella crassa Lamarck, F. limbata Sowerby, Scurria variabilis (Sowerby), Acanthopleura echinata (Bames) y Enoplochiton niger (Barnes).

Distribución: Dall (1909), desde Valparaíso, Chile a Mollendo, Perú; Marincovich (1973), desde Islas Chincha, Perú a Talcahuano, Chile.

Localidades: Asia, Pucusana, San Bartolo, Carquín. Se amplia el límite norte de la distribución geográfica hasta la localidad de Carquín (110 05'43'S)

Scurria variabilis (Sowerby, 1839)

(Figs $6-8$ )

Acmaea variabilis, Peña, 1970:155

Collisella variabilis, Marincovich, 1973:20 fig. 35

Valva de altura variable, de contorno irregular, redondeado a oval; con suaves costillas anchas y finas; color gris verdoso con los interespacios estrechos de color pardo oscuro, 0 con bandas pardas radiales; interior con el área central con manchas marrones irregulares; la impresión muscular y el área adyacente de color pardo amarillento; el margen de la conchilla de color azulado deja traslucir las bandas pardas extemas. Longitud, $33 \mathrm{~mm}$; ancho, 27 mm; altura, $9 \mathrm{~mm}$.

Observaciones: Común en el mediolitoral medio e inferior y en la franja infralitoral sobre la superficie de las rocas de orillas expuestas. En los ejemplares mas grandes las valvas están corroídas y es frecuente la epibiosis por Chthamalus scabrosus Darwin, y en menor grado por Scurria parasitica (Orbigny). Hallada en Pucusana (Isla Galápagos), sobre la superficie libre de la roca maciza expuesta, en la biocenosis de mitrlidos y por debajo.

Marincovich (1973) señala haber examinado sólo las conchillas del LACM y considera provisionalmente a esta especie como Collisella variabilis. El material colectado presenta un cordón branquial bien defino, por lo que no hay duda que pertenece al género Scurria.

Distribución: Dall (1909), toda la Provincia Peruana e Islas Galápagos: Marincovich (1973), Iquique y Valparaiso, Chile (collection LACM).

Localidades: Pucusana (Isla Galápagos).

Scurria zebrina (Lesson, 1830)

(Figs: $9-10)$

Collisella zebrina, Marincovich, 1973: 20, fig. 36.

Valva cónica, gruesa, alta y de base oval; la escultura consiste en costillas radiales prominentes, proyectadas ligeramente en el margen, y cruzadas por finas estrías de crecimiento; coloración verde oscura negruzca, en los interespacios, y grisácea en las costillas; con manchas triangulares mas claras o más oscuras, en los interespacios; interior blanco amulado, con el area central teñida de amarillento a marrón oscuro; margen de la conchilla con la misma coloración que el exterior. Longitud, $23 \mathrm{~mm}$; ancho, $17 \mathrm{~mm}$; altura, $12 \mathrm{~mm}$. ciza.

Observaciones: Escasa en el mediolitoral medio e inferior, sobre la superficie rocosa ma-

Aunque los ejemplares colectados son mas pequeños que los de Iquique, presentan un cordón branquial conformado por lóbulos prominentes que se retraen algo con la fijación.

Marincovich (1973) consideró tentativamente a esta especie dentro del genero Collisella, pero señala que los ejemplares de lquique poseen un cordón branquial consistente en una estructura tubular que presenta constricciones regularmente espaciadas. 
Debido a que nuestro material presenta un cordón branquial mejor definido, lo hemos incluido en el genero Scurria.

Distribución: Marincovich (1973), Iquique, Chile. Según este autor, en la colección del LACM hay ejemplares de esta especie procedentes de las Islas Chincha, Perú a Valparaíso, Chile; Dall (1909), desde Mollendo hasta la región Magallánica; Dell (1971) (Marincovich, 1973) la reporta en Tierra del Fuego.

Localidades: Asia, Pucusana y Ancón. Se amplía el límite norte de la distribución geográfica de esta especie hasta la latitud de Ancón (110 34'45'so.

\author{
Collisella Dall, 1871 \\ Collisella orbignyi (Dall, 1909)
}

(Figs. $11-12$ )

Acmaea orbignyi, Vegas, 1968: 4, Fig. 22

Acmaea orbignyi, Peña, 1970: 155; 1972: 39 , Fig. 12

Collisella orbignyi, Marincovich, 1973:20, fig. 34

Valva cónica de color pardo grisáceo; interior blanco con manchas marrones irregulares en el área central. Longitud, $29 \mathrm{~mm}$; ancho, $23 \mathrm{~mm}$; altura, $11 \mathrm{~mm}$.

Observaciones: Escasa en el mediolitoral superior y la franja supralitoral de orillas expuestas, sobre la superficie libre de las rocas; frecuente en el mediolitoral superior, en orillas protegidas, sobre las rocas cubiertas de algas. Algunos ejemplares presentan pequeños Chthamalus cirratus Darwin, epizoicos.

Distribución: Dall (1909), toda la Provincia Peruana, desde el Archipiélago Choros al Callao y las Islas Galápagos; Vegas (1968), Tortugas, Ancón, Pucusana, Laguna Grande; Peña (1972), de Trujillo a Tacna; Marincovich (1973) de Paita, Perú a Talcahuano, Chile. Según este autor, el reporte de las Islas Galápagos debe ser erróneo.

Localidades: Hallada en todos los lugares de estudio.

\title{
Collisella ceciliana (Orbigny, 1841)
}

(Fig. $13-14$ )

Collisella ceciliana, Marincovich, 1973:19, fig. 33

Valva delgada de contorno oval, ligeramente mas ancha posteriormente; escultura con suaves costillas radiales que se proyectan ligeramente en el margen; color externo verde oscuro amarillento moteado de gris con aspecto reticulado; costillas de color grisáceo, interespacios con fina estriación radial; interior azul verdoso, área central marrón con margen amarillento; margen interno con bandas marrones coincidentes con los interespacios externos; los ejemplares más grandes tienen el interior blanco, incluyendo la mayor parte del área central. Longitud, $22 \mathrm{~mm}$; ancho, $17 \mathrm{~mm}$; altura, $6 \mathrm{~mm}$.

Observaciones: Común en la franja supralitoral y el mediolitoral superior y medio, sobre la superficie de las rocas y en las grietas pequeñas; ejemplares pequeños y elevados viven epizoicos sobre Perumytilus purpuratus (Lamarck) y en menor grado sobre Semimytilus algosus (Gould); algunos ejemplares presentan pequeños Chthamalus cirratus Darwin, epizoicos. En Asia (Punta Lobería), Ancón y Chancaillo se encontraron ejemplares parasitados por una larva de Diptera.

Hay una gran variación en la altura de la conchilla, desde formas muy deprimidas hasta muy elevadas. En Pucusana se hallaron conchillas varadas de $26 \mathrm{~mm}$ de longitud.

Distribuci6n: Dall (1909), de Antofagasta a Valparaíso, Chile; Marincovich (1973), Iquique, Chile; Carcelles y Williamson (Marincovich, 1973), Estrecho de Magallanes, Tierra del Fuego, Sur de Patagonia e Islas Malvinas; Dell (Marincovich, 1973), Chile, entre los $42^{\circ}$ y $450 \mathrm{~S}$.

Según Marincovich (1973), en la colección del LACM hay ejemplares procedentes de Coquimbo y Valparaíso (Chile) y Pucusana, (Perú). 
Localidades: Asia, Pucusana, La Herradura, Ancón, Carquín, Chancafllo. Se amplía el limite norte de la distribución geográfica hasta la latitud de Chancamlo $\left(11^{\circ} 29^{\prime} 27^{\prime \prime} \mathrm{S}\right)$.

Collisella araucana (Orbigny. 1839)

(Fig. 15 - 16)

Collisella araucana, Marincovich, 1973: 18, fig. 32.

Valva de contorno oval con prominentes costillas radiales que se proyectan en el margen; costillas color gris claro, interespacios verde parduzco; interior blanquecino con el area central marrón oscuro y el margen ondulado con surcos que se corresponden con las costillas. Longitud, $19.5 \mathrm{~mm}$; ancho, $15 \mathrm{~mm}$; altura, $6 \mathrm{~mm}$.

Observaciones; Escasa en el mediolitoral, sobre las rocas; algunos ejemplares presentan pequeños Chthamalus cirratus Darwin, epizoicos.

Distribución: Marincovich (1973), Iquique a Viña del Mar, Chile e Islas Malvinas.(Orbigny, 1841). Según Marincovich, el reporte de Dall (1909) en Paita (Perú) requiere confirmación.

Localidades: Pucusana.

\section{AGRADECIMIENTOS}

Expreso mi reconocimiento al Doctor James H. Mclean y al Doctor Louie Marincovich, del Natural History Museum Los Angeles County, quienes gentilmente me enviaron sus publicaciones.

Asimismo, agradezco la colaboración del personal del Laboratorio de Zoología General de la Universidad Nacional Mayor de San Marcos, en la realización de los trabajos de campo.

\section{REFERENCIAS BIBLIOGRAFICAS}

DALL, W.H. 1909, Report on a collection of shells form Peru, with a summary of the littoral marine Mollusca of the Peruvian Zoological province. Proc. U.S.N. Mus, 37 (1704): 147-294, pls. 20-28.

KEEN, A. MYRA. 1960. Sea Shells of Tropical West America. Stanford Univ. Press, California. 624 pp., 1,709 figs., 10 pls.

1971. Sea Shells of Tropical West America. 2a. ed. Stanford Univ. Press, California. 1,064 pp., 3,325 figs., 22 pls.

MARINCOVICH, L 1973. Intertidal Mollusks of Iquique, Chile. Los Angeles Co. Mus. Nat. Hist. Sci. Bull. 16: 149, 102 figs.

McLEAN, J.H. 1969. Marine Shells of Southern California. Los Angeles Co. Mus. Nat. Hist., Sci. Ser. 24, Zool. 11: 1-104, 54 figs.

OLSSON, A.A. 1961. Mollusks of the tropical eastern Pacific. Paleontol. Res. Inst. Ithaca, New York. 574 pp., pls. $1-86$.

PAREDES, C. 1974. El modelo de zonación en la orilla rocosa del Departamento de Lima. Rev. Per. Bíol. 1 (2) : $166-191$.

PEÑA, G.M. 1970. Zonas de distribución de los Gasteorópodos Marinos del Perú. An. Cient. U.A., 8 (3-4): $153 \cdot 170$.

1972. Gasterópodos Marinos del Perú con descripción de dos nuevas especies. Tesis de Doctorado. Univ. Nac. Mayor de San Marcos. Lima, Perú, 342 pp., 200 figs.

VEGAS, M. 1968. Revision Taxonómica y Zoogeográfica de algunos gasterópodos y lamelibranquios marinos del Perú. An. Cient. U.A., 4 (1-2): 1-29, 53 figs. 


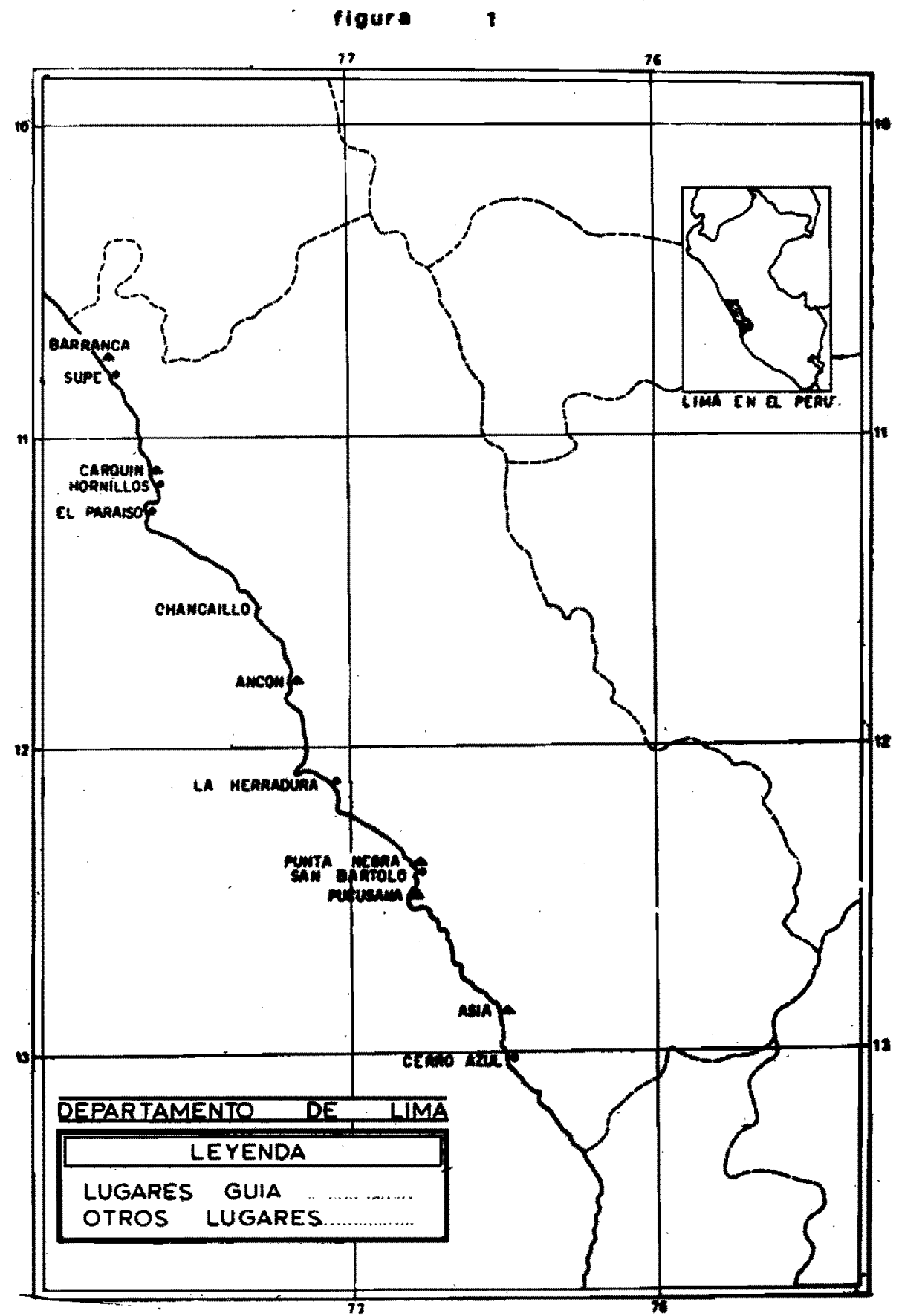



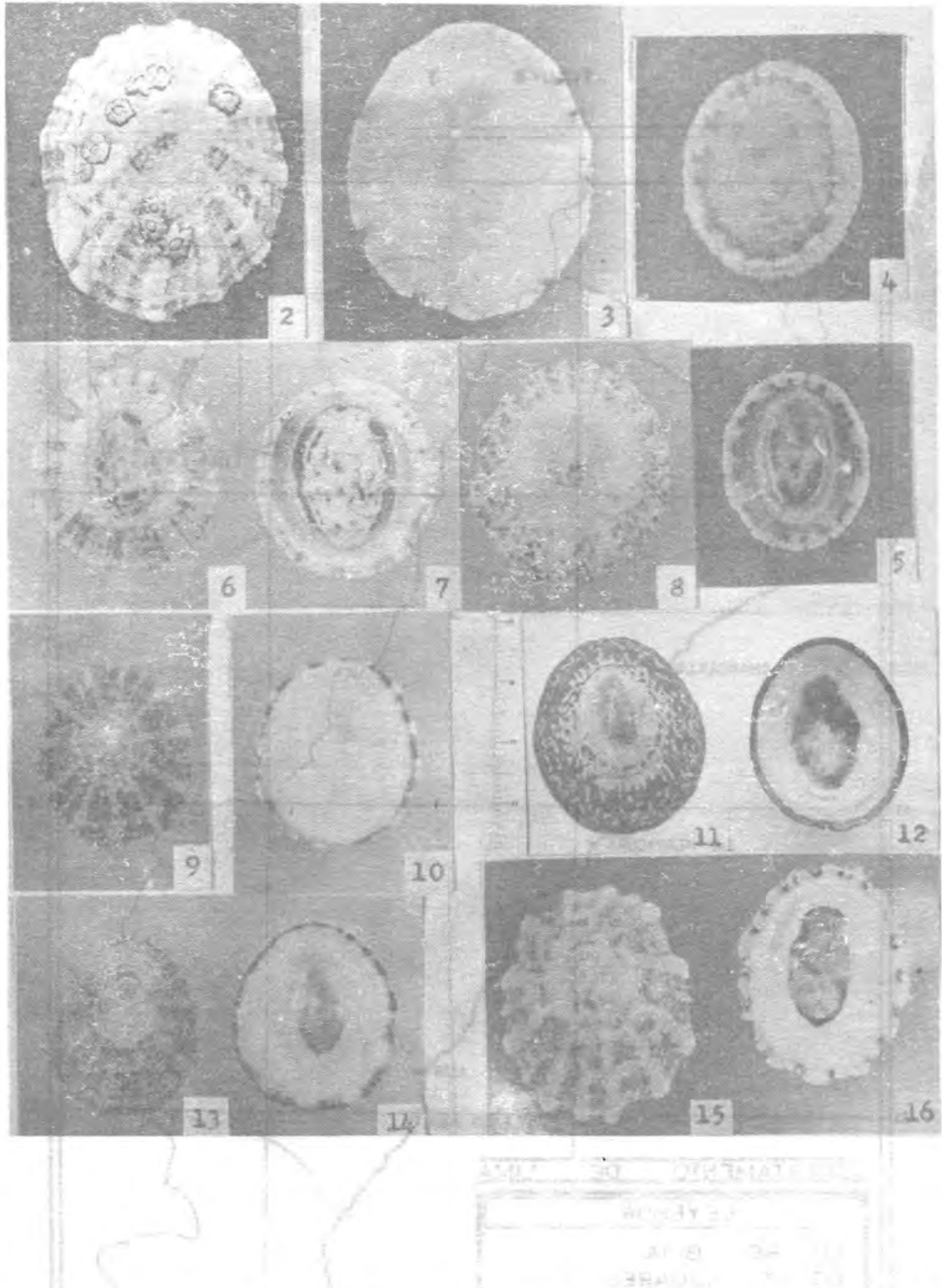

Figuras: 2-16. 2-3, Scurria viridula, longitud $39 \mathrm{~mm} ; 4-5$, S parasitica, longitud $15 \mathrm{~mm} ; 6-8$, S. variabilis, longitud $33 \mathrm{~mm} ; 9-10$, S. zebrina, longitud $23 \mathrm{~mm} ; 11-12$, Collisella orbignyi, longitud, $29 \mathrm{~mm} ; 13-14, C$. ceciliana, longitud $22 \mathrm{~mm} ; 15-16, C$. araucana, longitud $19.5 \mathrm{~mm}$. 